\title{
SELETIVIDADE, EFICIÊNCIA DE CONTROLE DE PLANTAS DANINHAS E PERSISTÊNCIA NO SOLO DE IMAZAMOX APLICADO NA CULTURA DO FEIJOEIRO ${ }^{1}$
}

\author{
TARCÍSIO COBUCCI ${ }^{2}$ e EDUARDO MACHADO ${ }^{3}$
}

\section{RESUMO}

O presente trabalho teve como objetivo avaliar a seletividade, o controle de plantas daninhas e a persistência no solo de imazamox aplicado na cultura do feijoeiro, durante os anos de 1995 a 1997. Nos experimentos de seletividade foram testados: imazamox (20, 30 e 40 g i.a./ha), imazamox + bentazon $(30+480 \mathrm{~g}$ i.a./ha), imazamox + fomesafen $(30+125 \mathrm{~g}$ i.a./ha) $\mathrm{e}$ testemunha nas cultivares Pérola, Jalo Precoce, Novo Jalo e Xamego. Os experimentos foram mantidos livres de plantas daninhas e foram avaliadas a fitotoxicidade visual e a produção de grãos. Nos experimentos de eficiência de controle de plantas daninhas foram testados: imazamox (20, 30 e 40 g i.a./ha), bentazon (480 g i.a./ha), fomesafen (125 e 250 g i.a./ha), imazamox + bentazon $(30+480$ g i.a./ha), imazamox + fomesafen $(30+125$ g i.a./ha $)$, bentazon / imazamox (480/30 g i.a/ha, aplicação seqüencial), fomesafen/imazmox (125/30 g i.a./ha, aplicação seqüencial), fomesafen/fomesafen (100/100 g i.a./ha, aplicação seqüencial) e testemunha. Foram avaliadas as porcentagens de controle de Euphorbia heterophylla e Bidens pilosa. Para o estudo da persistência de imazamox no solo, foram conduzidos experimentos em dois locais: Goiânia, GO (argiloso) e Jussara, GO (arenoso). Imazamox (40 g i.a./ha) causou injúrias à cultura do feijoeiro que acarretaram redução de $15 \%$ na produtividade (média dos experimentos). A mistura de imazamox e bentazon causou menor grau de injúrias no feijoeiro, obtendo-se ganhos de produtividade de $8 \%$ (médias dos experimentos). Imazamox mostrou-se ineficiente para o controle de Bidens pilosa, enquanto para Euphorbia heterophylla observou-se controle eficiente a partir da dose de 40 gi.a./ha. Aplicações seqüenciais de fomesafen/imazamox (125/30 g i.a/ha) apresentaram controle eficiente de Euphorbia heterophylla e Bidens pilosa. A persistência de imazamox no solo, para ambos os locais, foi maior em 1995 que 1996. Isto foi devido à maior umidade do solo em 1996. A sensibilidade das culturas sucedâneas aos resíduos de imazamox no solo foi, em ordem decrescente: sorgo, milho e arroz. O período entre a aplicação do herbicida e o plantio da cultura sucedânea (INP) variou de acordo com a sensibilidade das culturas aos resíduos de imazamox no solo e à sua persistência. Considerando ambos os locais e anos, o INP variou de 68 a 111 dias para milho, 78 a 139 dias para sorgo e 25 a 75 dias para o arroz.

Palavras chave: Phaseolus vulgaris, herbicidas, bioensaio, atividade residual.

\section{ABSTRACT \\ Selectivity, efficacy and soil persistence of imazamox applied to edible bean}

The present work evaluated the selectivity, efficacy and soil persistence of imazamox applyied to edible bean. The experiments were carried out from 1995 to 1997 . In the selectivity

\footnotetext{
${ }^{1}$ Recebido para publicação em 24/06/98 e na forma revisada em 11/09/99.

${ }^{2}$ Eng $^{\circ}$ Agro $^{\circ}$, Dr., Embrapa Arroz e Feijão. C.P. 179, CEP: 74001-970, Goiânia/GO.

${ }^{3}$ Graduando do Curso de Agronomia da Universidade Federal de Uberlândia. Av. Engenheiro Diniz, 1.178, CEP: 38401-136, Uberlândia/MG.
} 
experiments we tested: imazamox $(20,30$ e $40 \mathrm{~g} / \mathrm{ha})$, imazamox + bentazon $(30+480 \mathrm{~g} / \mathrm{ha})$, imazamox + fomesafen $(30+125 \mathrm{~g} / \mathrm{ha})$ and control with no herbicides with the varieties, Pérola, Jalo Precoce, Novo Jalo and Xamego. The selectivity experiments were maintained weed-free by hand, and the visual fitotoxicity and grain yield were evaluated. The efficacy experiments tested the effects of imazamox (20, 30 and $40 \mathrm{~g} / \mathrm{ha})$, bentazon (480 g/ha), fomesafen (125 and $250 \mathrm{~g} / \mathrm{ha})$ imazamox + bentazon $(30+480 \mathrm{~g} / \mathrm{ha})$, imazamox + fomesafen $(30+125 \mathrm{~g} / \mathrm{ha})$, bentazon/imazamox $\quad(480 / 30 \mathrm{~g} / \mathrm{ha}, \quad$ sequential application), fomesafen/imazamox $(125 / 30 \mathrm{~g} / \mathrm{ha}$, sequential application), fomesafen/fomesafen (100/100 g/ha, sequential application), on the control of Euphorbia heterophylla and Bidens pilosa. To study the persistence of imazamox in soil we conducted experiments in Goiânia (clay) and Jussara (loamy sand). Imazamox (40 g/ha) caused injury on common bean which meant a $15 \%$ decrease in grain yield (average of

\section{INTRODUÇ̃̃O}

O número de herbicidas recomendados para o controle de espécies de plantas daninhas de folhas largas em pós-emergência, na cultura do feijoeiro é bastante restrito. O bentazon é um composto de amplo espectro, não tendo, todavia, ação sobre algumas plantas daninhas de ocorrência freqüente, como Alternanthera tennela, Amaranthus ssp e Euphorbia heterophylla (Alcântara \& Carvalho, 1982; Brandão et al., 1982; Vidal et al., 1984). A absorção do bentazon ocorre essencialmente pelas folhas e pelos tecidos verdes, sendo menos intensa pelas raízes. A translocação é reduzida quando ele é absorvido pelas folhas e quando absorvido pelas raízes, verifica-se pequena movimentação acrópeta, pelo xilema. Nas espécies tolerantes, o produto é metabolizado por meio da formação de hidroxibentazon, com ruptura do anel heterocíclico.

$\mathrm{O}$ bentazon tem pka de 3,2 , o que lhe confere, em pHs normalmente encontrados nos experiments). The tank mix of imazamox and bentazon caused less injuries and improved grain yield by $8 \%$. Imazamox (40 g/ha) was inefficient to control Bidens pilosa while it was able to control Euphorbia heterophyla. Sequential application of fomesafen/imazamox (125/30 g i.a./ha) was efficient to control Bidens pilosa and Euphorbia heterophyla. The persistence of imazamox in soil, at both locations, was longer in 1995 than 1996. This was due to higher soil moisture content in 1996 than 1995. The rotational crops most sensitive to imazamox residues were, in decreasing order: sorghum, corn and rice. The period between herbicide application and rotational crop planting (INP) varied according to the sensitivity of that crop to the presence and persistence of herbicide residues in the soil. The INP for Imazamox $(40 \mathrm{~g} / \mathrm{ha})$ ranged from 68 to 111 days for corn, 78 to 139 days for sorghum, and 25 to 75 days for rice.

Key words: Phaseolus vulgaris, herbicide, bioassay, residual activity.

solos, a forma aniônica. Em pH neutro, em razão de sua alta solubilidade e de suas características aniônicas, a adsorção é menor, resultando em maior mobilidade (Abernathy \& Wax, 1973). Esse produto é adsorvido em solos orgânicos e, graças ao rápido processo de degradação microbiana, tem baixa persistência no solo. A dose recomendada para a cultura do feijoeiro varia de 0,72 a $0,96 \mathrm{~kg}$ i.a./ha, de acordo com o estádio de desenvolvimento das plantas daninhas (Alcântara \& Carvalho, 1982; Laca-Buendia \& Kakida, 1982).

O mecanismo de ação dos difeniléteres (fomesafen) sobre algas foi estudado, e verificouse que os carotenóides e, posteriormente, a clorofila são destruídos, ocorrendo formação de etanol e inibição do transporte do oxigênio da fotossíntese. De acordo com Weller \& Warren (1992), a interação da clorofila com os difeniléteres é explicada pela inativação da protoporfirinogênio oxidase (enzima da rota de síntese de clorofila). Com isso, forma-se a protoporfirina não-enzimática que, na presença de 
luz e $\mathrm{O}_{2}$, forma o oxigênio singleto, responsável pela peroxidação de lipídios, os quais levam à ruptura da membrana e ao vazamento de compostos celulares para os espaços intercelulares.

A protoporfirina não-enzimática não serve de substrato para a Mg-quelatase; dessa forma, a síntese de clorofila é reduzida. A facilidade de retenção de água pelas folhas (local da planta onde o produto é aplicado), a penetração foliar e o metabolismo estão relacionados com a seletividade dos difeniléteres a certas culturas. $\mathrm{O}$ primeiro sintoma de fitotoxicidade do fomesafen caracteriza-se pela embebição de água, seguida de necrose dos tecidos verdes, seis horas após a aplicação sob luz. Esses sintomas são observados na cultura do feijoeiro, na dose de $0,25 \mathrm{~kg}$ i.a./ha, verificando-se, entretanto, completa recuperação da cultura dez dias após (Laca-Buendia \& Kakida, 1982).

O imazamox é um herbicida do grupo das imidazolinonas e que inibem a enzima sintetase do ácido acetohidróxido (ALS) que participa na formação de três aminoácidos: leucina, valina e isoleucina (Anderson \& Hibberd, 1985). O composto é predominantemente absorvido pelas folhas e translocado. Os primeiros sintomas caracterizam-se pela clorose nas folhas mais novas, seguida de necrose dos meristemas apicais. As imidazolinonas podem persistir no solo e causar problemas para culturas sucedâneas (Peterson \& Arnold, 1985; Ritter et al., 1988; Barnes et al., 1989; Loux et al., 1989; Monks \& Banks, 1991; Wixson e Shaw, 1992; Krausz et al., 1994). Silva et al. (1995) relataram que imazamox (50 g i.a./ha), aplicado na soja, não apresentou efeito fitotóxico residual em milho e sorgo plantados 90 dias após a aplicação do herbicida. Entretanto, poucas informações são disponíveis a respeito da degradação do herbicida e de seus efeitos em culturas sucedâneas em condições de plantio de feijão de inverno, além da seletividade para a cultura do feijoeiro e sua eficiência no controle de plantas daninhas.

O objetivo deste trabalho foi avaliar a seletividade e eficiência do herbicida imazamox aplicado em diferentes cultivares de feijoeiro e o controle de plantas daninhas e determinar os efeitos de seus resíduos no solo sobre culturas sucedâneas.

\section{MATERIAL E MÉTODOS}

\section{Seletividade}

Foram conduzidos sete experimentos na Embrapa Arroz e Feijão, em Santo Antônio de Goiás, GO, em Latossolo Vermelho-Escuro, sendo quatro com a cultivar Pérola (inverno/95, inverno/96, seca/97 e inverno/97), um com Xamego (inverno/97), um com Jalo Precoce (inverno/97) e um com Novo Jalo (inverno/97). Algumas características químicas e granulométricas do solo estão apresentadas na Tabela 1. Efetuou-se a semeadura das culturas do feijoeiro, cultivar Pérola, em 26/6/95, 14/6/96, 2/2/97 e 9/6/97; cultivar Xamego, 6/6/97, Jalo Precoce, 9/6/97 e Novo Jalo 9/6/97, no espaçamento de $0,40 \mathrm{~m}$ entre linhas com 16 sementes por metro à profundidade média de $0,05 \mathrm{~m}$. A adubação de base foi realizada com $450 \mathrm{~kg} / \mathrm{ha}$ na fórmula 5:30:15 no sulco de plantio. Adubação nitrogenada em cobertura foi realizada 25 dias após a emergência, com $60 \mathrm{~kg} \mathrm{~N} / \mathrm{ha}$. Efetuou-se irrigação de acordo com a necessidade da cultura. Os tratamentos foram imazamox (20, 30 e 40 g i.a./ha), imazamox + bentazon $(30+480$ g i.a./ha), imazamox + fomesafen $(30+125$ g i.a./ha) e testemunha, no delineamento em blocos casualisados. Nos tratamentos com herbicidas foi adicionado Agral, na proporção de $0,125 \% \mathrm{v} / \mathrm{v}$. Todos os tratamentos foram mantidos livres da competição com plantas daninhas com a realização de duas capinas manuais. Os herbicidas foram aplicados quando o feijoeiro apresentava-se no estádio de dois a três trifólios, empregando equipamento costal pressurizado com gás carbônico $\left(\mathrm{CO}_{2}\right)$, provido de barra com quatro pontas de jato plano marca TeeJet DG 80.015 VS, espaçadas entre si de $0,50 \mathrm{~m}$, totalizando $2,0 \mathrm{~m}$ de largura efetiva. $\mathrm{O}$ equipamento foi operado a $40 \mathrm{lb} / \mathrm{pol}^{2}$, empregando água como diluente e volume de aplicação correspondente a $200 \mathrm{l} / \mathrm{h}$ a. As 
avaliações de fitotoxicidade à cultura foram realizadas de forma visual aos 14 e 21 dias após a aplicação, empregando a escala percentual na qual zero $(0 \%)$ representa sem sintoma de fitotoxicidade aparente e $100 \%$, morte da planta. A produção de grãos, foi avaliada corrigindo a umidade para $13 \%$. Os dados de produção foram submetidos à análise de variância, sendo as médias comparadas pelo teste de Tukey a $5 \%$ de probabilidade.

\section{Eficiência de controle de plantas daninhas}

Foram conduzidos cinco experimentos na Embrapa Arroz e Feijão, em Santo Antônio de Goiás, GO, em Latossolo Vermelho-Escuro, nos anos de 1995, 1996 e 1997. Efetuou-se semeadura da cultura do feijoeiro $(6 / 6 / 95,4 / 2 / 96,9 / 6 / 96$, 10/2/97, 5/6/97), cultivar Pérola, no espaçamento de $0,4 \mathrm{~m}$ entre linhas com 16 sementes por metro, à profundidade de $0,05 \mathrm{~m}$. A adubação de base foi realizada com $450 \mathrm{~kg} / \mathrm{ha}$ na fórmula 5:30:15 no sulco de plantio. A adubação nitrogenada em cobertura foi realizada 25 dias após a emergência com $60 \mathrm{~kg} \mathrm{~N} / \mathrm{ha}$. Efetuou-se irrigação de acordo com a necessidade da cultura. Características químicas e granulométricas do solo estão apresentadas na Tabela 1 . O delineamento experimental empregado foi o de bloco ao acaso, com 11 tratamentos e quatro repetições, sendo a unidade experimental de $16 \mathrm{~m}^{2}$ (2x8 m). Os tratamentos foram imazamox $(20,30$
40 g i.a./ha), bentazon (480 g i.a./ha), fomesafen (125 e 250 gi.a./ha) imazamox + bentazon $(30+480$ g i.a./ha), imazamox + fomesafen $(30+125$ g i.a./ha), bentazon/ imazamox (480/30 g i.a/ha, aplicação seqüencial), fomesafen/imazamox (125/30 g i.a./ha, aplicação seqüencial), fomesafen/fomesafen $\quad(100 / 100$ g i.a./ha, aplicação seqüencial) e testemunha. Nos tratamentos com imazamox e fomesafen foi adicionado Agral na proporção de $0,125 \%$ v/v e com bentazon isolado 1 l/ha de Assit. Os herbicidas foram aplicados quando o feijoeiro apresentava-se no estádio de dois a três trifólios e as plantas daninhas com duas a quatro folhas e, nas aplicações seqüenciais, a primeira aplicação foi realizada com o feijoeiro no primeiro trifólio, e a segunda aplicação dez dias após. Na aplicação dos produtos utilizou-se o mesmo equipamento do experimento de seletividade com a mesma pressão e volume de aplicação. As avaliações de controle de plantas daninhas foram realizadas de forma visual aos 28 dias após a aplicação, empregando a escala percentual na qual zero $(0 \%)$ representa nenhum controle e $100 \%$, controle total. Devido à baixa produtividade de alguns experimentos, a produção de grãos não foi considerada. As espécies daninhas avaliadas nos experimentos e a população média foram: Euphorbia heterophylla, 250, 45, 350, 46 e $100 \mathrm{pl} / \mathrm{m}^{2}$ e Bidens pilosa, 32, $25,17,36$ e $54 \mathrm{pl} / \mathrm{m}^{2}$, no inverno/95, seca/96, inverno/96, seca/97 e inverno/97, respectivamente. e

TABELA 1. Algumas características químicas e granulométricas de amostras do solo. Santo Antônio de Goiás, GO.

\begin{tabular}{cccccc}
\hline $\mathrm{Ph}$ & M.O. & $\mathrm{CTC}$ & Argila & Silte & Areia \\
\hline$\%$ & $\mathrm{Cmol}_{\mathrm{c}} \mathrm{Kg}^{-1}$ & $-----{ }^{-}$ & & & \\
5,6 & 1,6 & 8,2 & 52,5 & 18,5 & 29,0 \\
\hline
\end{tabular}

Ca e $\mathrm{Mg}$ extraídos em $\mathrm{KCl} 1 \mathrm{~N}, \mathrm{Al}$ extraído em $\mathrm{NaOH}, \mathrm{K}, \mathrm{P}$ extraídos em solução de Mehlich $\left(\mathrm{HCl} 0,5 \mathrm{~N}+\mathrm{H}_{2} \mathrm{SO}_{4}\right.$ $0,025 \mathrm{~N})$; matéria orgânica determinada pelo método de Walkley Black. 
Para a avaliação de efeitos sinergísticos, aditivos ou antagonísticos das misturas dos herbicidas e das aplicações seqüenciais foi empregado o método de Colby (1967). Para cada porcentagem de controle observado na mistura dos herbicidas ou aplicações seqüenciais, calculou-se o valor esperado expresso pela seguinte equação:

$$
\mathrm{E}=\mathrm{X}+[\mathrm{Y}(100-\mathrm{X}) / 100]
$$

onde, $\mathrm{X}=$ porcentagem observada de controle do herbicida $\mathrm{A}, \mathrm{Y}=$ porcentagem de controle do herbicida B. Se o valor observado fosse superior, igual ou inferior ao esperado, o efeito seria considerado sinergístico, aditivo ou antagonístico, respectivamente. Nash (1981) relatou como desvantagem deste método a não consideração da variação do acaso. Para solucionar este problema os valores das diferenças (observadas/esperadas) foram submetidos à análise de variância. Para testar se a diferença média era nula empregou-se o teste $\mathrm{t}$.

\section{Persistência no solo}

Quatro experimentos foram conduzidos em Goiânia, GO, em Latossolo Vermelho-Escuro e em Jussara, GO, em Areia Quartzoza, nos anos de 1995 e 1996. O delineamento experimental foi o de blocos casualizados, com quatro repetições. Os tratamentos consistiram de aplicações do herbicida imazamox (40 g i.a./ha, em 1995; 40 e $80 \mathrm{~g}$ i.a./ha em 1996) e testemunha. O tamanho das parcelas foi de $2 \times 8 \mathrm{~m}$, sendo a área útil de $1 \times 6 \mathrm{~m}$.

Em ambas as localidades, o solo foi preparado com uma aração e duas gradagens. A cultivar Aporé foi semeada em 17/6/95 e 31/5/96 em Goiânia e 12/7/95 e 15/7/96 em Jussara, com espaçamento entre linhas de $0,5 \mathrm{~m}$ e com adubação de base de $300 \mathrm{~kg} / \mathrm{ha}$ da fórmula 4-3016. A adubação nitrogenada em cobertura foi realizada com $100 \mathrm{~kg} \mathrm{~N} / \mathrm{ha}$, aos 25 dias dias após a emergência.

Os tratamentos foram aplicados em 13/7/95 e 5/7/96 em Goiânia, e 10/8/95 e 20/8/96 em Jussara, com um pulverizador pressurizado a
$\mathrm{CO}_{2}$ com barra de quatro bicos (TJ 80015) espaçados a $0,5 \mathrm{~m}$ e vazão de $200 \mathrm{l} / \mathrm{ha}$ a $40 \mathrm{lb} / \mathrm{pol}^{2}$. Todas as parcelas foram mantidas livres de plantas daninhas. As características químicas e granulométricas dos solos e dados de precipitação estão listados nas Tabelas 2 e 3.

Dez amostras de solo por parcela $(8 \mathrm{~cm}$ de diâmetro e $10 \mathrm{~cm}$ de profundidade) foram coletadas em ambas localidades aproximadamente $0,50,75,100,125$ e 150 dias após a aplicação do produto, para realização de bioensaios em casa de vegetação. As amostras foram secas ao ar, passadas em peneira de $10 \mathrm{~mm}$ e misturadas para formação de uma amostra composta, na qual $750 \mathrm{~g}$ de solo foram colocados em vaso de $1 \mathrm{~kg}$. Cinco sementes de milho, Zea mays (AG 603), arroz, Oriza sativa (Maravilha) e sorgo, Sorghum bicolor (Cargill C-42), todos pré-germinados por $36 \mathrm{~h}$, com comprimento de radícula de $3+/-1 \mathrm{~mm}$ foram semeadas separadamente em cada vaso a 2 $\mathrm{cm}$ de profundidade. $\mathrm{O}$ solo foi saturado com água e mantido próximo da capacidade de campo. Aos 13 dias após semeadura, as plantas foram colhidas, secadas por $36 \mathrm{~h}$ em estufa a $72^{\circ} \mathrm{C}$ e pesadas. Com base na produção da matéria seca correspondente aos tratamentos herbicidas e da testemunha foram calculadas as porcentagens de crescimento (em relação à testemunha) para cada cultura, ano e dose dos herbicidas, as quais foram submetidas à análise de regressão em função de dias.

Para determinar a concentração dos herbicidas no solo, amostras de solo não tratadas de ambas as localidades foram tratadas separadamente com imazamox para obter as concentrações de $0,5,10,20,30,50,100,150$, 200 e 300 ppb. Cinco sementes de sorgo (Cargill C-42) pré-germinadas foram semeadas e, após a colheita das plantas, foram obtidas as porcentagens de crescimento (em relação à testemunha). As equações de regressão da curva padrão obtidas estão apresentadas na Tabela 4, onde $\mathrm{Y}$ é a porcentagem de crescimento de sorgo em relação à testemunha aos 13 dias após a semeadura e $\mathrm{X}$ é a concentração de imazamox no solo. 
TABELA 2. Algumas características químicas e granulométricas de amostras do solo de Goiânia e Jussara nos experimentos de persistência de imazamox no solo.

\begin{tabular}{ccccccc}
\hline Local & $\mathrm{pH}$ & M.O. & CTC & Argila & Silte & Areia \\
\hline & & $\%$ & $\mathrm{Cmol}_{\mathrm{c}} \mathrm{Kg}^{-1}$ & $-----{ }^{2}$ & & \\
Goiânia & 5,4 & 2,1 & 7,98 & 43,0 & 21,5 & 35,5 \\
Jussara & 6,2 & 0,7 & 4,70 & 10,5 & 3,0 & 86,5 \\
\hline
\end{tabular}

Ca e Mg extraídos em $\mathrm{KCl} 1 \mathrm{~N}, \mathrm{Al}$ extraído em $\mathrm{NaOH}, \mathrm{K}, \mathrm{P}$ extraídos em solução de Mehlich ( $\mathrm{HCl} 0,5 \mathrm{~N}+\mathrm{H}_{2} \mathrm{SO}_{4} 0,025 \mathrm{~N}$ ); matéria orgânica determinada pelo método de Walkley Black.

TABELA 3. Dados de precipitação de água $(\mathrm{mm})$ nos experimentos de persistência de imazamox no solo em Goiânia e Jussara, 1995 e 1996.

\begin{tabular}{|c|c|c|c|c|}
\hline \multirow[t]{2}{*}{ Tempo (DAA)* } & \multicolumn{2}{|c|}{ Goiânia } & \multicolumn{2}{|c|}{ Jussara } \\
\hline & 1995 & 1996 & 1995 & 1996 \\
\hline $0-15$ & 47,5 & 115,0 & 53,2 & 81,2 \\
\hline $16-30$ & 63,8 & 98,0 & 68,3 & 92,0 \\
\hline $31-45$ & 74,8 & 90,2 & 64,9 & 88,0 \\
\hline $46-60$ & 84,5 & 80,5 & 100,2 & 93,2 \\
\hline $61-75$ & 18,9 & 81,2 & 90,3 & 80,3 \\
\hline Média/dia & 3,86 & 6,19 & 5,02 & 5,79 \\
\hline $76-90$ & 129,4 & 46,4 & 51,2 & 33,0 \\
\hline $91-105$ & 40,1 & 34,8 & 85,0 & 100,2 \\
\hline $106-120$ & 36,0 & 141,5 & 92,1 & 87,0 \\
\hline $121-135$ & 106,8 & 29,2 & 82,3 & 75,2 \\
\hline $136-150$ & 32,5 & 72,9 & 90,7 & 102,0 \\
\hline Média/dia & 4,99 & 4,32 & 5,35 & 5,29 \\
\hline
\end{tabular}

*Dias após aplicação dos herbicidas

TABELA 4. Equações padrão para determinação da concentração de imazamox no solo em Goiânia e Jussara.

\begin{tabular}{lccc}
\hline Local & Cultura & \multicolumn{2}{c}{ Equação padrão } \\
\hline Goiânia & & & \\
Jussara & Sorgo & $\mathrm{y}=97,5-5,99 \mathrm{X}^{1 / 2}$ & $\mathrm{R}^{2}=0,91$ \\
\hline
\end{tabular}

$\mathrm{y}=$ porcentagem de crescimento de plantas de sorgo em relação à testemunha

$\mathrm{x}=$ concentração de herbicida no solo $(\mathrm{ppb})$ 
As porcentagens de crescimento estimadas para as amostras de sorgo do campo foram substituídas na equação da respectiva curva padrão para determinar a concentração do herbicida no solo. O limite de detecção para o bioensaio foi de $5 \mathrm{ppb}$. As concentrações de herbicida no solo, para cada ano e local, foram submetidas à análise de regressão em função de dias para obter a curva de degradação destes produtos.

Com as equações de porcentagem de crescimento em função de dias para cada cultura, ano e local, foram obtidos os valores dos dias necessários para igualar a testemunha $(100 \%)$ e, a partir daí, os valores foram substituídos em sua respectiva equação da curva de degradação dos herbicidas no solo para obter a concentração do herbicida nesta época.

A meia-vida dos herbicidas para cada local e ano foi calculada a partir da concentração inicial (intercepto do y) usando a curva de degradação dos herbicidas.

Nas análises de regressão, foram ajustados os modelos polinomiais, sendo os coeficientes testados pelo teste $\mathrm{t}$, a $5 \%$ de probabilidade. $\mathrm{O}$ modelo escolhido foi o que obteve todos os coeficientes, ou pelo menos o de maior grau, significativos, sendo $\mathrm{R}^{2}$ o mais alto possível e o independente da regressão não-significativo.

\section{RESULTADOS E DISCUSSÃO}

\section{Seletividade}

Com relação à fitotoxicidade dos tratamentos herbicidas, verifica-se na Tabela 5 que o herbicida imazamox causou danos visuais à cultura do feijoeiro aos 14 dias após aplicação (DAA), sendo mais severos na dose de $40 \mathrm{~g}$ i.a./ha. Os danos à cultura do feijoeiro se caracterizaram pelo amarelecimento das folhas mais jovens, e nas cultivares precoces (Jalo Precoce e Novo Jalo) houve também redução do porte das plantas na dose de $40 \mathrm{~kg}$ i.a./ha. Nesta dose, a produção do feijão, em todos os experimentos, foi menor em relação à testemunha (15\% na média dos experimentos) (Tabela 6), sendo que nos experimentos com Jalo Precoce, Novo Jalo e Pérola/inverno 96, a redução apresentou-se significativa. $\mathrm{Na}$ dose de $30 \mathrm{~g}$ i.a./ha a redução média foi de $4 \%$, entretanto não se observou efeito significativo em nenhum dos experimentos.

A mistura de bentazon (480 g i.a./ha) e imazamox (30 g i.a./ha) resultou, em todos os experimentos, em menor fitotoxicidade ao feijoeiro aos 14 DAA (Tabela 5), em relação à aplicação isolada de imazamox (30 g i.a./ha). Neste tratamento a produtividade do feijão foi superior em $8 \%$ na média dos experimentos, apesar de não significativo em nenhum deles. Bentazon tem demonstrado ser um antagônico à fitotoxicidade de haloxyfop (Gerwick, 1988), sethoxydin (Rhodes \& Coble, 1984; Jordan et al., 1990) e paraquat (Evans et al., 1988; Wehtje et al., 1992). Em todos os casos o antagonismo tem sido atribuído à redução de absorção foliar. Provavelmente, a diminuição da fitotoxicidade da mistura bentazon e imazamox pode estar relacionada com a redução da absorção foliar de imazamox pelo feijoeiro. Estudos complementares, entretanto, devem ser conduzidos. A mistura de imazamox com fomesafen não causou diminuição da fitotoxicidade ao feijoeiro em relação a aplicação isolada de imazamox (Tabela 5); conseqüentemente, não houve diferença na produtividade (médias dos experimentos) (Tabela $6)$.

\section{Eficiência de controle de plantas daninhas}

Na média dos cinco experimentos, para o controle de Euphorbia heterophylla os melhores tratamentos foram a aplicação de imazamox a $40 \mathrm{~g}$ i.a./ha; e a aplicação sequiencial de fomesafen (125 g i.a./ha) e imazamox (30 g i.a./ha). As misturas imazamox+bentazon $(30+480$ g i.a./ha $)$ e imazamox+fomesafen apresentaram valores não significativos da diferença da porcentagem de controle observada e esperada (OBS-ESP) (Tabela 7) indicando comportamento aditivo, ou seja, não se verificou efeito antagônico da mistura. 
TABELA 5. Valores médios de porcentagens de fitotoxicidade à cultura do feijoeiro aos 14 e 21 dias após aplicação. Santo Antônio de Goiás, 1995, 1996 e 1997.

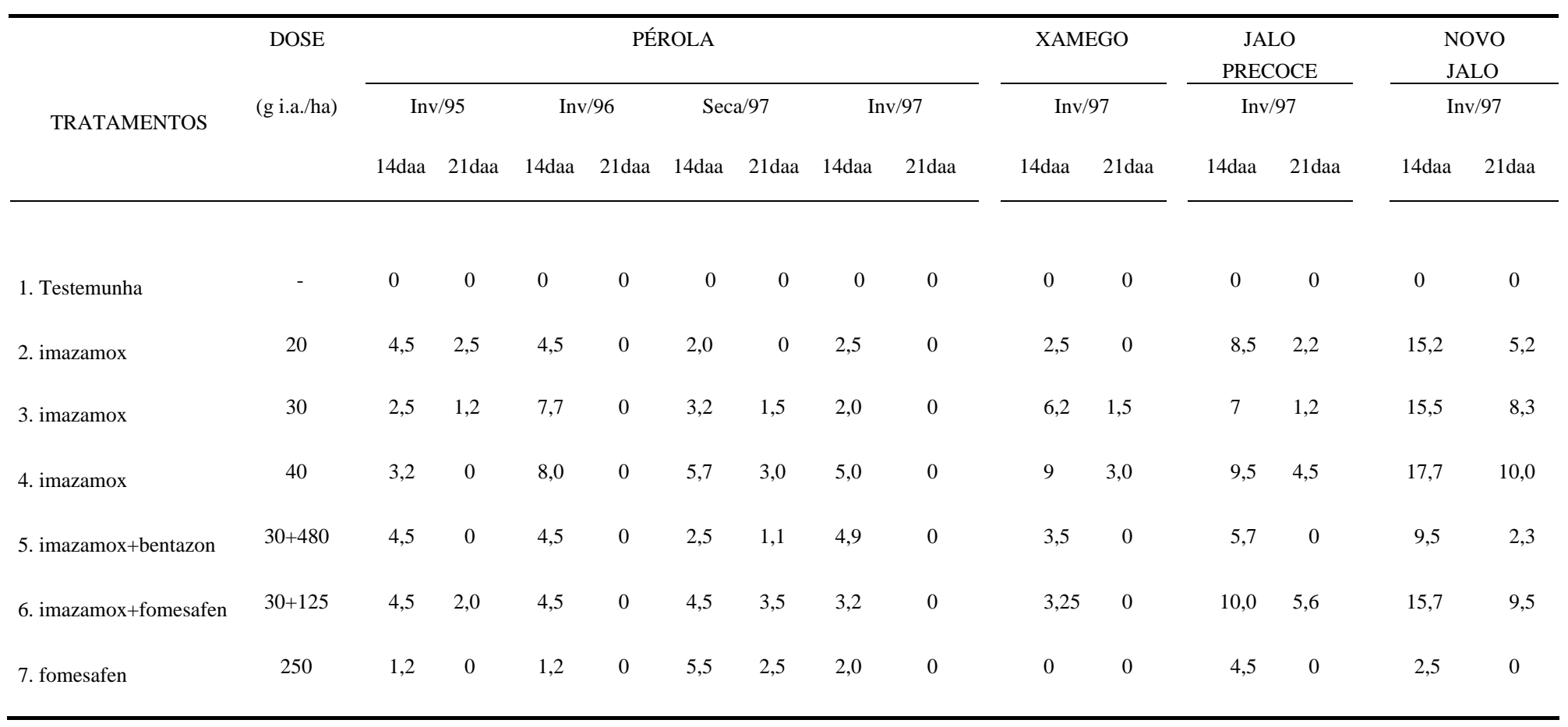


TABELA 6. Valores médios de porcentuais de produção de grãos em relação à testemunha. Santo Antônio de Goiás. 1995, 1996 e 1997.

\begin{tabular}{|c|c|c|c|c|c|c|c|c|c|}
\hline \multirow{2}{*}{ TRATAMENTOS } & \multirow{2}{*}{$\begin{array}{c}\text { DOSE } \\
\text { (gia/ha) }\end{array}$} & \multicolumn{4}{|c|}{ PÉROLA } & \multirow{2}{*}{$\begin{array}{c}\text { XAMEGO } \\
\text { Inv/97 }\end{array}$} & \multirow{2}{*}{$\begin{array}{c}\text { JALO PRECOCE } \\
\text { Inv/97 }\end{array}$} & \multirow{2}{*}{$\begin{array}{c}\text { NOVO JALO } \\
\text { Inv/97 }\end{array}$} & \multirow[t]{2}{*}{ Média } \\
\hline & & Inv/95 & Inv/96 & Seca/97 & Inv/97 & & & & \\
\hline 1. Testemunha & - & $100 \mathrm{a}$ & $100 \mathrm{a}$ & $100 \mathrm{a}$ & $100 \mathrm{a}$ & $100 \mathrm{a}$ & $100 \mathrm{ab}$ & $100 \mathrm{a}$ & 100 \\
\hline 2. imazamox & 20 & $103 \mathrm{a}$ & $86 \mathrm{a}$ & $101 \mathrm{a}$ & $90 \mathrm{a}$ & $110 \mathrm{a}$ & $93 \mathrm{ab}$ & 95 a & 96 \\
\hline 3. imazamox & 30 & $102 \mathrm{a}$ & 87 a & 109 a & $93 \mathrm{a}$ & $101 \mathrm{a}$ & $95 \mathrm{ab}$ & $90 \mathrm{a}$ & 96 \\
\hline 4. imazamox & 40 & 99 a & $63 \mathrm{~b}$ & 96 a & 91 a & $86 \mathrm{a}$ & $86 \mathrm{~b}$ & $77 \mathrm{~b}$ & 85 \\
\hline 5. imazamox+bentazon & $30+480$ & $110 \mathrm{a}$ & $100 \mathrm{a}$ & $120 \mathrm{a}$ & 98 a & 107 a & $106 \mathrm{ab}$ & 93 a & 104 \\
\hline 6. imazamox + fomesafen & $30+125$ & $93 \mathrm{a}$ & $104 \mathrm{a}$ & $110 \mathrm{a}$ & $93 \mathrm{a}$ & $98 \mathrm{a}$ & $102 \mathrm{ab}$ & $74 \mathrm{~b}$ & 96 \\
\hline 7. fomesafen & 250 & $111 \mathrm{a}$ & 99 a & 119 a & 105 a & $118 \mathrm{a}$ & $108 \mathrm{a}$ & $108 \mathrm{a}$ & 109 \\
\hline C.V. & & 17,58 & 18,98 & 13,45 & 15,32 & 14,34 & 8,76 & 10,2 & \\
\hline \multicolumn{2}{|c|}{ Produção Testemunha (kg/ha) } & 2785 & 3238 & 962 & 3533 & 2324 & 3255 & 2938 & \\
\hline
\end{tabular}

Médias seguidas pela mesma letra na coluna não diferem significativamente pelo teste de Tukey a 5\% de probabilidade. 
TABELA 7. Valores médios da porcentagem de controle de Euphorbia heterophylla aos 28 dias de aplicação. Santo Antônio de Goiás, GO, 1995, 1996 e 1997.

\begin{tabular}{|c|c|c|c|c|c|c|c|c|}
\hline \multirow[t]{2}{*}{ Tratamentos } & \multirow{2}{*}{$\begin{array}{c}\text { Dose } \\
\text { (g i.a./ha) } \\
\end{array}$} & \multicolumn{7}{|c|}{ Euphorbia heterophylla } \\
\hline & & Inv/95 & Seca/96 & Inv/96 & Seca/97 & Inv/97 & Média & Obs-Esp ${ }^{a}$ \\
\hline 1. Testemunha & - & 0 & 0 & 0 & 0 & 0 & 0 & \\
\hline 2. imazamox & 20 & 70 & 55 & 50 & 60 & 70 & 61,0 & \\
\hline 3. imazamox & 30 & 86 & 81 & 75 & 83 & 81 & 81,2 & \\
\hline 4. imazamox & 40 & 98 & 91 & 94 & 88 & 90 & 92,2 & \\
\hline 5. bentazon & 480 & 0 & 0 & 0 & 0 & 0 & 0 & \\
\hline 6. fomesafen & 125 & 51 & 30 & 30 & 50 & 40 & 40,2 & \\
\hline 7. fomesafen & 250 & 81 & 70 & 80 & 78 & 85 & 78,8 & \\
\hline 8. imazamox+bentazon & $30+480$ & 89 & 81 & 75 & 83 & 83 & 82,2 & $-1,0^{\mathrm{ns}}$ \\
\hline 9. imazamox+fomesafen & $30+125$ & 92 & 87 & 76 & 86 & 85 & 85,2 & $-3,2^{\mathrm{ns}}$ \\
\hline 10. bentazon/imazamox & $480 / 30$ & 85 & 85 & 72 & 80 & 75 & 79,4 & $-1,8^{\text {ns }}$ \\
\hline 11. fomesafen/imazamox & $125 / 30$ & 93 & 93 & 96 & 90 & 94 & 93,2 & $4,7 *$ \\
\hline 12. fomesafen/fomesafen & $100 / 100$ & 85 & 90 & 80 & 90 & 85 & 86,0 & \\
\hline \multicolumn{2}{|c|}{ População média testemunha (pl/m²) } & 250 & 45 & 350 & 46 & 100 & 158,2 & \\
\hline \multicolumn{2}{|l|}{ Desvio padrão } & & & & & & & 1,73 \\
\hline \multicolumn{2}{|l|}{ Diferença mínima para zero } & & & & & & & 3,77 \\
\hline
\end{tabular}

${ }^{\mathrm{a}}$ Porcentagem de controle observada menos a esperada.

*Significativo no nível de 5\% de probabilidade pelo Teste de Tukey.

O mesmo resultado foi observado para a aplicação seqüencial de bentazon/imazamox. Entretanto, nenhum destes tratamentos foi eficiente no controle de Euphorbia heterophylla. A aplicação seqüencial de fomesafen/imazamox, além de se mostrar eficiente no controle desta planta daninha, apresentou OBS-ESP significativo e positivo, indicando que houve sinergismo no controle de Euphorbia heterophylla.

Para o controle de Bidens pilosa, imazamox mostrou-se ineficiente mesmo na dose de $40 \mathrm{~g}$ i.a./ha (Tabela 8). Imazamox aplicado em mistura ou em aplicação seqüencial com bentazon ou fomesafen apresentou-se eficiente, apesar de se verificar efeito antagônico da mistura ou da aplicação seqüencial (valores OBS-ESP significativos e negativos).
Considerando as condições experimentais, a aplicação de fomesafen (125 g i.a./ha), realizada no estádio de duas folhas das plantas daninhas, e dez dias após a aplicação de imazamox (30 g i.a./ha), parece ser a mais indicada para o controle simultâneo de Euphorbia heterophylla e Bidens pilosa. Estudos futuros devem ser realizados com a aplicação de bentazon + imazamox (aplicados após fomesafen, seqüencial), pois os dados indicam que a mistura causa menor injúria às plantas do feijoeiro, podendo resultar em aumento na produtividade.

\section{Persistência no solo}

As curvas de degradação e a meia-vida de imazamox para cada ano, local e dose estão 
apresentadas na Tabela 9. Observa-se que tanto em Goiânia como em Jussara a meia-vida de imazamox foi maior em 1996 em relação a 1995. A maior taxa de degradação de imazamox em 1996, foi, provavelmente, devida a maiores lâminas de água aplicadas naquele ano (Tabela 3). Em 1995 a precipitação (irrigação) média diária aplicada nos primeiros 75 dias após a aplicação dos herbicidas, nos ensaios de Goiânia e Jussara, foi 3,86 e 5,02, respectivamente, contra 6,19 e 5,79 em 1996. A umidade do solo tem mostrado influência na respiração microbiana e, conseqüentemente, na taxa de degradação de herbicidas do grupo das imidazolinonas (Basham \& Levy, 1987). Além disso, em solos com maior teor de umidade, as moléculas de água competem com mais sucesso que as moléculas do herbicida pelos pontos de adsorção nos colóides do solo. Assim, as moléculas do produto tendem a permanecer livres na solução do solo, onde estarão sujeitas à lixiviação, absorção pelas plantas e microrganismos (Van Scoyoc \& Ahlrischs, 1992). Goetz et al. (1990); Basham \& Lavy (1987) verificaram em seus estudos que imazethapyr e imazaquin apresentaram maior persistência em solos com menores teores de umidade.

As culturas sucedâneas responderam diferentemente aos resíduos de imazamox no solo. Observa-se na Tabela 10 que a cultura do sorgo apresentou-se mais susceptível, tolerando imazamox no solo abaixo de 5 ppb (CLHS) (média de local e dose). Para o milho, a CLHS foi de 10,2 ppb, seguido pelo arroz (39,9 ppb).

TABELA 8. Valores médios da porcentagem de controle de Bidens pilosa aos 28 dias após a aplicação. Santo Antônio de Goiás, GO, 1995, 1996 e 1997.

\begin{tabular}{|c|c|c|c|c|c|c|c|c|}
\hline \multirow[t]{2}{*}{ Tratamentos } & \multirow{2}{*}{$\begin{array}{c}\text { Dose } \\
\text { (g i.a./ha) }\end{array}$} & \multicolumn{7}{|c|}{ Bidens pilosa } \\
\hline & & Inv/95 & Seca/96 & Inv/96 & Seca/97 & Inv/97 & Média & Obs-Esp $^{a}$ \\
\hline 1. Testemunha & - & 0 & 0 & 0 & 0 & 0 & 0 & \\
\hline 2. imazamox & 20 & 51 & 40 & 30 & 60 & 50 & 46,2 & \\
\hline 3. imazamox & 30 & 66 & 56 & 53 & 70 & 62 & 61,4 & \\
\hline 4. imazamox & 40 & 70 & 78 & 76 & 80 & 68 & 74,4 & \\
\hline 5. bentazon & 480 & 90 & 95 & 80 & 97 & 83 & 89,0 & \\
\hline 6. fomesafen & 125 & 87 & 90 & 80 & 80 & 92 & 85,8 & \\
\hline 7. fomesafen & 250 & 90 & 93 & 93 & 94 & 93 & 92,6 & \\
\hline 8. imazamox+bentazon & $30+480$ & 90 & 93 & 83 & 96 & 82 & 88,8 & $-6,7 *$ \\
\hline 9. imazamox+fomesafen & $30+125$ & 92 & 90 & 90 & 93 & 94 & 91,8 & $-2,7 *$ \\
\hline 10. bentazon/imazamox & $480 / 30$ & 90 & 90 & 83 & 95 & 84 & 88,4 & $-7,2 *$ \\
\hline 11. fomesafen/imazamox & $125 / 30$ & 92 & 93 & 90 & 98 & 90 & 92,6 & $-2,0 *$ \\
\hline 12. fomesafen/fomesafen & $100 / 100$ & 95 & 98 & 92 & 98 & 93 & 95,2 & \\
\hline \multicolumn{2}{|c|}{ População média testemunha $\left(\mathrm{pl} / \mathrm{m}^{2}\right)$} & 32 & 25 & 17 & 36 & 34 & 32,8 & \\
\hline \multicolumn{2}{|l|}{ Desvio padrão } & & & & & & & 0,86 \\
\hline \multicolumn{2}{|l|}{ Diferença mínima para zero } & & & & & & & 1,87 \\
\hline
\end{tabular}

${ }^{a}$ Porcentagem de controle observada menos a esperada

* Significativo ao nível de $5 \%$ de probabilidade pelo Teste de Tukey 
TABELA 9. Equações de degradação no solo e meia-vida de imazamox em Goiânia e Jussara, 1995 e 1996.

\begin{tabular}{|c|c|c|c|c|}
\hline Local & Ano & $\begin{array}{c}\text { Dose } \\
\left(\mathrm{g} \mathrm{ai} \mathrm{ha}^{-1}\right)\end{array}$ & Equação de degradação no solo & $\begin{array}{c}\text { Meia-vida } \\
\text { (Dias) }\end{array}$ \\
\hline Goiânia & 95 & 40 & $\mathrm{y}^{\mathrm{a}}=64,5-5,47 \mathrm{X}^{\mathrm{b} 1 / 2} \quad \mathrm{R}^{2}=0,83$ & 34,7 \\
\hline Goiânia & 96 & 40 & $y=77,3-8,40 X^{1 / 2} \quad R^{2}=0,96$ & 21,1 \\
\hline Goiânia & 96 & 80 & $\mathrm{y}=125,0-13,5 \mathrm{X}^{1 / 2} \mathrm{R}^{2}=0,96$ & 21,4 \\
\hline Jussara & 95 & 40 & $\mathrm{y}=64,5-6,14 \mathrm{X}^{1 / 2} \quad \mathrm{R}^{2}=0,89$ & 27,5 \\
\hline Jussara & 96 & 40 & $y=66,6-6,87 X^{1 / 2} \quad R^{2}=0,88$ & 23,4 \\
\hline Jussara & 96 & 80 & $y=108,7-10,4 X^{1 / 2} R^{2}=0,93$ & 27,3 \\
\hline
\end{tabular}

TABELA 10. Equações de porcentagem de crescimento (em relação à testemunha) das culturas de milho, sorgo e arroz em função de dias após aplicação de imazamox; concentração limite de herbicida no solo (CLHS) e intervalo de dias para plantio das culturas sucedâneas (INP), Goiânia, GO e Jussara, GO, 1995 e 1996.

\begin{tabular}{|c|c|c|c|c|c|c|c|}
\hline \multirow{3}{*}{$\begin{array}{r}\text { Cultura } \\
\text { Milho }\end{array}$} & \multirow{2}{*}{$\begin{array}{r}\text { Local } \\
\text { Goiânia }\end{array}$} & \multirow{2}{*}{$\begin{array}{c}\text { Ano } \\
95\end{array}$} & \multirow{2}{*}{$\begin{array}{c}\text { Dose } \\
40\end{array}$} & \multicolumn{2}{|c|}{ Equação crescimento } & \multirow{2}{*}{$\begin{array}{c}\begin{array}{c}\text { CLHS* } \\
(\mathrm{ppb})\end{array} \\
10\end{array}$} & \multirow{2}{*}{$\begin{array}{r}\text { INP*** } \\
99\end{array}$} \\
\hline & & & & $\mathrm{y}=45,6+5,45 \mathrm{X}^{1 / 2}$ & $\mathrm{R}^{2}=0,96$ & & \\
\hline & Goiânia & 96 & 40 & $y=46,8+1,159 X-0,0055 X^{2}$ & $\mathrm{R}^{2} 0,96$ & 19,3 & 68 \\
\hline & Goiânia & 96 & 80 & $y=40,1+1,363 X-0,00679 X^{2}$ & $\mathrm{R}^{2}=0,87$ & 16,1 & 65 \\
\hline & Jussara & 95 & 40 & $y=27,8+6,8 x^{1 / 2}$ & $\mathrm{R}^{2}=0,96$ & 5,1 & 111 \\
\hline & Jussara & 96 & 40 & $\mathrm{y}=27,2+7,7 \mathrm{X}^{1 / 2}$ & $\mathrm{R}^{2}=0,97$ & 6,2 & 88 \\
\hline & Jussara & 96 & 80 & $y=25,4+7,29 X^{1 / 2}$ & $\mathrm{R}^{2}=0,98$ & 5,0 & 105 \\
\hline & & & & & Média & 10,2 & 89 \\
\hline \multirow[t]{7}{*}{ Sorgo } & Goiânia & 95 & 40 & $y=39,7+5,11 X^{1 / 2}$ & $\mathrm{R}^{2}=0,76$ & $<5$ & 139 \\
\hline & Goiânia & 96 & 40 & $y=43,4+6,38 X^{1 / 2}$ & $\mathrm{R}^{2}=0,94$ & $<5$ & 78 \\
\hline & Goiânia & 96 & 80 & $\mathrm{y}=30,0+7,72 \mathrm{X}^{1 / 2}$ & $\mathrm{R}^{2}=0,87$ & $<5$ & 82 \\
\hline & Jussara & 95 & 40 & $y=32,6+7,12 X^{1 / 2}$ & $\mathrm{R}^{2}=0,90$ & 6,38 & 89 \\
\hline & Jussara & 96 & 40 & $y=32,7+6,62 X^{1 / 2}$ & $\mathrm{R}^{2}=0,90$ & $<5$ & 101 \\
\hline & Jussara & 96 & 80 & $\mathrm{y}=18,3+7,8 X^{1 / 2}$ & $\mathrm{R}^{2}=0,90$ & $<5$ & 109 \\
\hline & & & & & Média & $<5$ & 99 \\
\hline \multirow[t]{7}{*}{ Arroz } & Goiânia & 95 & 40 & $y=27,4+9,63 X^{1 / 2}$ & $\mathrm{R}^{2}=0,97$ & 23,5 & 56 \\
\hline & Goiânia & 96 & 40 & $y=37,8+2,91 X^{1 / 2}$ & $\mathrm{R}^{2}=0,97$ & 35,3 & 25 \\
\hline & Goiânia & 96 & 80 & $y=20,7+1,97 X-0,00811 X^{2}$ & $\mathrm{R}^{2}=0,99$ & 28,5 & 51 \\
\hline & Jussara & 95 & 40 & $y=36,3+7,33 X^{1 / 2}$ & $\mathrm{R}^{2}=0,88$ & 11,3 & 75 \\
\hline & Jussara & 96 & 40 & $y=39,6+7,13 X^{1 / 2}$ & $\mathrm{R}^{2}=0,81$ & 8,3 & 72 \\
\hline & Jussara & 96 & 80 & $\mathrm{y}=28,4+7,8 \mathrm{X}^{1 / 2}$ & $\mathrm{R}^{2}=0,73$ & 13,3 & 84 \\
\hline & & & & & Média & 39,9 & 60 \\
\hline
\end{tabular}

*Concentração dos herbicidas no solo para que as culturas sucedâneas não apresentem efeito depressivo dos herbicidas.

**Intervalo de dias para o plantio das culturas sucedâneas (dias necessários para alcançar a CLHS). 
Observa-se que, para todas as culturas, as CLHS, em Jussara, foram menores que em Goiânia (Tabela 10). Todos os herbicidas, quando em contato com o solo, são adsorvidos em algum grau, e sua atividade é reduzida na proporção direta da quantidade adsorvida. As moléculas adsorvidas ficam em um estado passivo, não disponíveis para os processos biológicos, físicos e químicos, até que um processo de dessorção ocorra. Neste caso, as moléculas ou os íons dos herbicidas adsorvidos serão deslocados dos pontos de adsorção, retornando à solução do solo, onde estarão sujeitos a processos biológicos (absorção por plantas, microrganismos). Provavelmente, em Jussara, devido aos menores teores de argila e matéria orgânica (Tabela 2), houve menor adsorção dos herbicidas no solo, ficando estes disponíveis para a absorção pelas plantas. Conseqüentemente, menores concentrações dos herbicidas no solo foram necessárias para que as plantas sucedâneas não apresentassem efeitos depressivos dos resíduos do herbicida no solo.

Os intervalos de dias necessários para o plantio das culturas sucedâneas (INP) variaram em função das diferentes taxas de degradação dos produtos e susceptibilidade das culturas aos resíduos do herbicida. Considerando as duas localidades e os dois anos, o INP para imazamox (40 g i.a./ha) variou de 68 a 111 dias para o milho, 78 a 139 dias para o sorgo e 25 a 75 dias para o arroz.

Considerando que o plantio das culturas sucedâneas após a colheita do feijão é feito aproximadamente 75 dias após a aplicação do produto, a probabilidade de injúria no sorgo, causada por resíduos de imazamox (40 g i.a./ha) é alta, enquanto para o arroz é baixa. Para o milho, injúria é possível sob certas condições ambientais (baixa umidade do solo e alto conteúdo de argila e matéria orgânica), entretanto parece ser baixa em condições de alta precipitação (em Goiânia $6,19 \mathrm{~mm} \mathrm{dia}^{-1}$.

\section{LITERATURA CITADA}

ALBERNATHY, J.R., WAX, L.M. Bentazon mobility and adsorption in twelve Illinois soils. Weed Sci., v.21, n.3, p.224-227, 1973.

ALCÂNTARA, E.N., CARVALHO, D.A. Controle de plantas daninhas. Inf. Agropec., v.8, n.90, p.30-32, 1982.

ANDERSON, P.C., HIBBERD R.A. Evidence for the interaction of an imidazolinone herbicide with leucine, valine and isoleucine metabolism. Weed Sci., v.33, p.479-484, 1985.

BARNES, C.J., GOETZ, A.J., LAVY, T.L. Effects of imazaquin residues on cotton (Gossypium hirsutum). Weed Sci., v.37, p.820-824, 1989.

BASHAM, G.W., LAVY, T.L. Microbial and photolytic dissipation of imazaquin in soil. Weed Sci., v.35, p.865-870, 1987.

BRANDÃO, M., LACA-BUENDIA, J.P., GAVILANES, M.L. Principais plantas daninhas no Estado de Minas Gerais. Inf. Agropec., v.8, n.87, p.18-32, 1982.

COLBY, S.R. Calculating synergistic and antagonistic responses of herbicide combinations. Weed Sci., v.15, p.20-22, 1967.

COLBY, S.R., BARNES, J.W. Fomesafen-New selective herbicide for post-emergence broadleaf weed control in soybean. Weed Abstract, v.33, p.7, 1984.

COLBY, S.R. Fomesafen, a breakthorugh in postemergence soybean weed control. Weed Abstract, v.32, p.288, 1983.

EVANS, J.R., TURNER, J.C., GOURD, D.R., McKEMIE, T.E. Interaction of bentazon and paraquat for peanut weed control. Southern Weed Science Society Proceedings, v.41, p.98, 1988. 
GERWICK, C.B. Potential mechanism for bentazon antagonism with haloxyfop. Weed Sci., v.36, p.286-280, 1988.

GOETZ, A.J., LAVY, T.L., GBUR, E.E. Degradation and field persistence of imazethapyr. Weed Sci., v.38, p.421-428, 1990.

JORDAN, D.L., YORK, A.C., CORBIN, F.T. Effect of ammonium sulfate and bentazon on sethoxydim adsorption. Weed Technol., v.3, p.674-677, 1990.

KRAUSZ, R.F., KAPUSTA, G., MATTHEWS, J.L. Soybean (Glycine max) and rotational crop response to PPI chlorimuron, clomazone, imazaquin, and imazethapyr. Weed Technol., v.8, p.224-230, 1994.

LACA-BUENDIA, J.P., KAKIDA, J. Comportamento de herbicidas pósemergentes na cultura do feijão, sob irrigação no norte de Minas Gerais. In: CONGRESSO BRASILEIRO DE HERBICIDAS E ERVAS DANINHAS, 14., 1982, Campinas. Resumos... Campinas, SBHPD, 1982. p.31.

LOUX, M.M., LIEBL, R.A., SLIFE, F.W. Adsorption of imazaquin and imazethapyr on soils, sediments, and selected adsorbents. Weed Sci., v.37, p.712-718, 1989.

MONKS, C.D., BANKS, P.A. Rotational crops response to chlorimuron, clomazone, and imazaquin applied the previous year. Weed Sci., v.39, p.629-633, 1991.

NASH, R.G. Phytotoxic interactions sudies techniques for evaluation and presentation of results. Weed Sci., v.29, n.2, p.147-155, 1981.

PETERSON, M.A., ARNOLD, W.E. Response of rotational crops to soil residues of chlorsulfuron. Weed Sci., v.34, p.131-136, 1985.

RHODES, G.N., COBLE, H.D. Influence of bentazon on absorption and translocation of sethoxydim is goosegras. Weed Sci., v.32, p.595-597, 1984.

RITTER, R.L., HARRIS, T.C., KAUFMAN, L.M. Chlorsulfuron and metsulfuron residues on double-cropped soybeans (Glycine max). Weed Technol., v.2, p.49-52, 1988.

SILVA, A.A., FERREIRA, F.A., BRITO, S.A., SANTOS, J.G.M. Efeito residual de Imazamox and Imazethapyr em Latossolo Roxo. In: CONGRESSO BRASILEIRO DA CIÊNCIA DAS PLANTAS DANINHAS, 20., 1995, Florianópolis. Resumos... Florianópolis, SBCPD, 1995. p.326.

VAN SCOYOC, G.E., AHLRICHS, J.L. Fate of herbicides in soils. In: PURDUE UNIVERSITY (West Lafayette). Herbicide action course. West Lafayette, 1992. p.407438 .

VIDAL, R.A., VEDOATO, R.A., WILES, J.P., WHITE, J.G. Uso de fomesafen para controle pós-emergente de plantas daninhas latifoliadas em soja. In: CONGRESSO BRASILEIRO DE HERBICIDAS E PLANTAS DANINHAS, 15., 1984, Belo Horizonte. Resumos... Belo Horizonte, SBHPD, 1984. p.90.

WEHTJE, G., WILCUT, J.W., McGUIRE, J.A. Influence of bentazon on the phytotoxicity of paraquat to penauts (Arachis hypogaea) and associated weeds. Weed Sci., v.40, p.90-95, 1992.

WELLER, S.C., WARREN, G.F. Diphenyletheres and oxadiazon. In: PURDUE UNIVERSITY (West Lafayette). Herbicide action course. West Lafayette, 1992. p.113120 .

WIXSON, M.B., SHAW, R. Effects of soilapplied AC 263, 222 on crops rotated with soybean (Glycine max). Weed Technol., v.6, p.276-279, 1992. 